\title{
Baseline investigation of Saudi seashore water concentrations along the Gulf of Aqaba
}

\author{
M. E. Elgohary ${ }^{1} \&$ S. M. Alaseri ${ }^{2}$ \\ ${ }^{I}$ Department of Industrial Engineering, \\ King Abdul Aziz University at Rabigh, Saudi Arabia \\ ${ }^{2}$ Faculty of Science, Tabuk University, Saudi Arabia
}

\begin{abstract}
This investigation aims to measure the elemental concentrations of sea water samples from the Gulf of Aqaba along the Saudi seashore. Seventy elements were investigated using the Inductively Coupled Plasma Mass Spectrometry (ICP-MS) technique. Sixty samples were collected starting from Ad Dorah city at the national border between Saudi Arabia and Jordan to Ra's Al-Sheikh Humayed at the south of Aqaba Gulf. Elemental concentrations of major, trace, toxic, and radioactive sources in this area are measured. The results represent a database for many vital areas of interest.
\end{abstract}

Keywords: ICP-MS, elemental analysis, sea water, Gulf of Aqaba, trace elements, concentrations.

\section{Introduction}

Although the Gulf of Aqaba region has remained relatively free of pollution [1], the environment is currently under increasing threat from a wide range of human activities. Also, marine-based activities such as shipping and oil exploitation are becoming a significant source of marine pollution in this Gulf. Except for this region, many national and international investigations have been carried out to the Red Sea water [1-8].

This work is part of a research project funded by Tabuk University to investigate this region. This work is dedicated to map the concentrations of Red Sea water along the Gulf of Aqaba of the Saudi Arabia seashore.

Inductively Coupled Plasma Mass Spectrometry (ICP-MS) will be used to measure the elemental concentration in this study. The technique was 
commercially introduced in 1983 and has gained general acceptance because of its superior detection capabilities [9].

\section{History and development of ICP-MS}

The major components of a modern Inductively Coupled Plasma Mass Spectrometry (ICP-MS) can be traced directly back to the work done during the 1960s and 1970s [10-13]. Dr Alan Gray of Applied Research Laboratories in Luton, UK, conducted much of the early research work that led to the commercial development of ICP-MS instrumentation [9]. Another important publication by Houk et al. in 1980 demonstrated the possibilities offered by the ICP-MS technique [14].

Although the early ICP-MS systems were expensive, large, complex, had limited automation and tended to suffer from significant signal drift, the obvious benefits of such multi-elements technique with low limits of detection and a simple mass spectrometric data output (including isotope ratio information) led to acceptance of the fledgling technique, particularly among those involved in research and geological applications. Due to many improvements, ICP-MS has become a widely used tool [15-19]. It is a flexible technique that offers many advantages over more traditional techniques for elemental analysis.

\section{Red Sea and Gulf of Aqaba}

The Gulf of Aqaba is located to the east of the Sinai Peninsula and west of the Arabian mainland [20]. This and the Gulf of Suez are the two gulfs extending from the northern portion of the Red Sea. It reaches a maximum depth of $1,850 \mathrm{~m}$ in its central basin. The Gulf measures 24 kilometers at its widest point and stretches some 160 kilometers long. Unfortunately, water renewal in the Red Sea is slow, and exchange with the ocean takes approximately 200 years for the entire sea.

Although the Red Sea is known for its natural beauty, there are major industries in the Red Sea region include oil industries, manufacturing industries, tourism, fisheries and oil transport. Besides, treated or untreated sewage effluents from plants, cargo vessels, tour boats and ferries have damaged marine life in certain areas inside the Gulf of Aqaba.

\section{Work aim, objectives, and targets}

The aim of this study is to investigate the state of pollution of the Gulf of Aqaba along the Saudi seashore. The objectives of this work are to:

1. Measure the levels of potential heavy metals, major components, radiation sources, and some trace elements in the sea water of the samples.

2. Shed some light on the most prominent contaminants of the marine resources in the region, as well as its possible implications for human health if any. 
3. Collect, review, and arrange the results that obtained during the study to serve as baseline data for further follow-up studies in this region.

This investigation covers seventy elements of periodic table. Table 1 illustrates these elements by their common used symbols.

Table 1: The following list illustrates seventy elements under investigation in Red sea water samples.

\begin{tabular}{|c|c|c|c|c|c|c|c|c|c|c|c|c|c|}
\hline $\mathrm{Ag}$ & $\mathrm{Al}$ & $\mathrm{As}$ & $\mathrm{Au}$ & $\mathrm{B}$ & $\mathrm{Ba}$ & $\mathrm{Be}$ & $\mathrm{Bi}$ & $\mathrm{Br}$ & $\mathrm{Ca}$ & $\mathrm{Cd}$ & $\mathrm{Ce}$ & $\mathrm{Cl}$ & $\mathrm{Co}$ \\
\hline $\mathrm{Cr}$ & $\mathrm{Cs}$ & $\mathrm{Cu}$ & $\mathrm{Dy}$ & $\mathrm{Er}$ & $\mathrm{Eu}$ & $\mathrm{Fe}$ & $\mathrm{Ga}$ & $\mathrm{Gd}$ & $\mathrm{Ge}$ & $\mathrm{Hf}$ & $\mathrm{Hg}$ & $\mathrm{Ho}$ & $\mathrm{In}$ \\
\hline $\mathrm{K}$ & $\mathrm{La}$ & $\mathrm{Li}$ & $\mathrm{Lu}$ & $\mathrm{Mg}$ & $\mathrm{Mn}$ & $\mathrm{Mo}$ & $\mathrm{Na}$ & $\mathrm{Nb}$ & $\mathrm{Nd}$ & $\mathrm{Ni}$ & $\mathrm{P}$ & $\mathrm{Pb}$ & $\mathrm{Pd}$ \\
\hline $\mathrm{Pr}$ & $\mathrm{Pt}$ & $\mathrm{Rb}$ & $\mathrm{Re}$ & $\mathrm{Rh}$ & $\mathrm{Ru}$ & $\mathrm{S}$ & $\mathrm{Sb}$ & $\mathrm{Sc}$ & $\mathrm{Se}$ & $\mathrm{Si}$ & $\mathrm{Sm}$ & $\mathrm{Sn}$ & $\mathrm{Sr}$ \\
\hline $\mathrm{Ta}$ & $\mathrm{Tb}$ & $\mathrm{Th}$ & $\mathrm{Ti}$ & $\mathrm{Tl}$ & $\mathrm{Tm}$ & $\mathrm{U}$ & $\mathrm{V}$ & $\mathrm{W}$ & $\mathrm{Ta}$ & $\mathrm{Y}$ & $\mathrm{Yb}$ & $\mathrm{Zn}$ & $\mathrm{Zr}$ \\
\hline
\end{tabular}

\section{Sampling and calibration}

Sixty water samples were collected during February 2011. The samples were taken from the seashore water. The region of interest is illustrated throughout the black dashed line shown in figure 1. Samples were collected in $200 \mathrm{ml}$ plastic bottles manufactured for chemical laboratories. Samples were numbered from 1 up to 60 according to site location number. The numbering started from the north at Ad Dorah city until the city of Ra's Al-Sheikh Humid. The geographical locations of the samples are listed in table 2 with the errors of GPS readings and the regions where the samples were taken from.

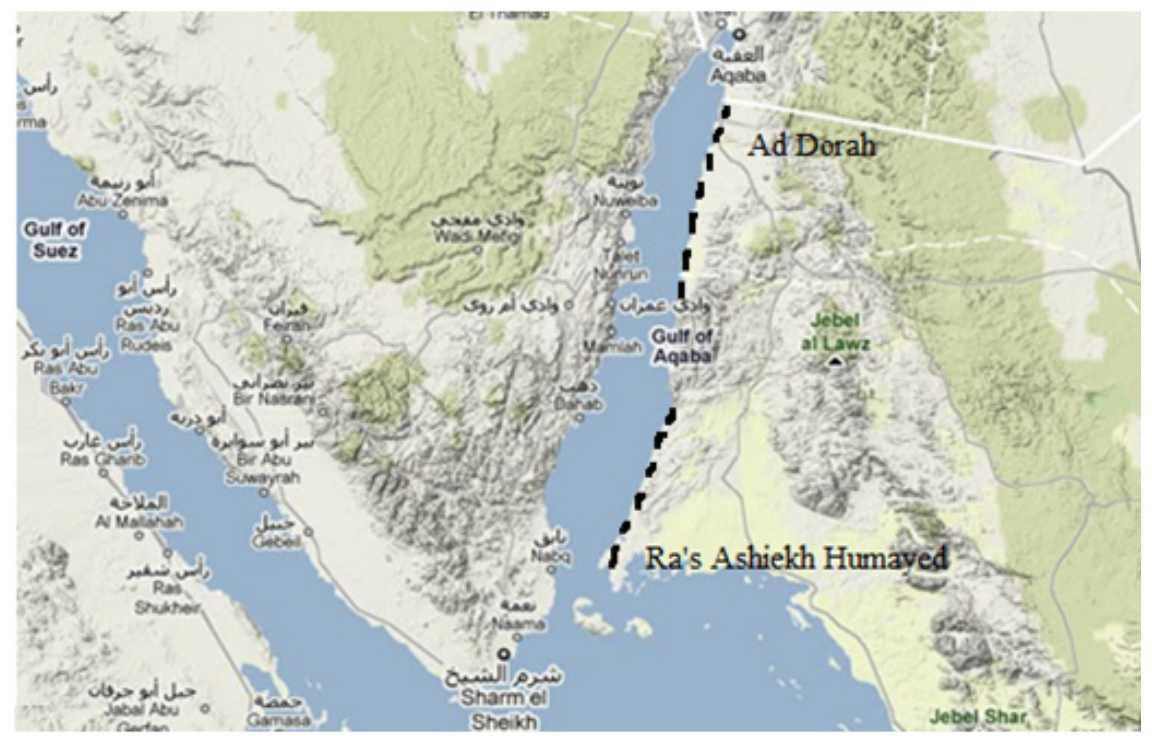

Figure 1: The Gulf of Aqaba region is shown. The black dashed line illustrates the path along which the sixty samples were collected. 
Table 2: Geographical locations and identification codes of the water samples.

\begin{tabular}{|c|c|c|c|c|}
\hline \multirow[t]{2}{*}{ Sample ID } & \multicolumn{2}{|c|}{ Geographical location } & \multirow{2}{*}{$\begin{array}{l}\text { error } \\
( \pm \mathrm{m})\end{array}$} & \multirow[t]{2}{*}{ Reference site } \\
\hline & North & East & & \\
\hline 1 & 29.3380 & 34.95000 & 4 & \multirow{5}{*}{ Ad Dorah } \\
\hline 2 & 29.3311 & 34.94893 & 6 & \\
\hline 3 & 29.3230 & 34.94503 & 5 & \\
\hline 4 & 29.31512 & 34.94786 & 4 & \\
\hline 5 & 29.30630 & 34.95104 & 5 & \\
\hline 6 & 29.29901 & 34.94946 & 6 & \multirow{7}{*}{ Haql } \\
\hline 7 & 29.30039 & 34.93801 & 5 & \\
\hline 8 & 29.28727 & 34.92873 & 4 & \\
\hline 9 & 29.27790 & $34 . .93108$ & 4 & \\
\hline 10 & 29.26712 & 34.93493 & 4 & \\
\hline 11 & 29.25267 & 34.93960 & 2 & \\
\hline 12 & 29.24146 & 34.94175 & 3 & \\
\hline 13 & 29.23410 & 34.93177 & 3 & \multirow{15}{*}{ Al Humaydah } \\
\hline 14 & 29.22940 & 34.91946 & 3 & \\
\hline 15 & 29.21668 & 34.91261 & 3 & \\
\hline 16 & 29.19475 & 34.90820 & 3 & \\
\hline 17 & 29.18811 & 34.89871 & 3 & \\
\hline 18 & 29.18366 & 34.89539 & 3 & \\
\hline 19 & 29.16452 & 34.89496 & 4 & \\
\hline 20 & 29.14950 & 34.89251 & 3 & \\
\hline 21 & 29.13983 & 34.88197 & 3 & \\
\hline 22 & 29.12753 & 34.87969 & 3 & \\
\hline 23 & 29.12304 & 34.88006 & 3 & \\
\hline 24 & 29.10978 & 34.87532 & 4 & \\
\hline 25 & 29.09291 & 34.87386 & 3 & \\
\hline 26 & 29.07555 & 34.87169 & 3 & \\
\hline 27 & 29.07318 & 34.87271 & 3 & \\
\hline 28 & 29.07020 & 34.87265 & 2 & \multirow{3}{*}{ Ra's Dabr } \\
\hline 29 & 29.06911 & 34.87179 & 4 & \\
\hline 30 & 29.06551 & 34.86944 & 3 & \\
\hline 31 & 28.44526 & 34.75785 & 3 & \multirow{9}{*}{ Maqna } \\
\hline 32 & 28.43604 & 34.75777 & 3 & \\
\hline 33 & 28.43244 & 34.75546 & 3 & \\
\hline 34 & 28.42028 & 34.75175 & 4 & \\
\hline 35 & 28.41032 & 34.74424 & 3 & \\
\hline 36 & 28.40022 & 34.74049 & 2 & \\
\hline 41 & 28.34919 & 34.72308 & 2 & \\
\hline 42 & 28.33982 & 34.71671 & 3 & \\
\hline 43 & 28.33192 & 34.71280 & 3 & \\
\hline
\end{tabular}


Table 2: $\quad$ Continued.

\begin{tabular}{|l|l|l|l|l|}
\hline 44 & 28.32101 & 34.70717 & 2 & \multirow{2}{*}{ Sharm Al Ishsh } \\
\hline 45 & 28.30841 & 34.70168 & 3 & \\
\hline 46 & 28.29068 & 34.69466 & 2 & \\
\hline 47 & 28.25229 & 34.67577 & 3 & \\
\hline 48 & 28.24627 & 34.67844 & 2 & \\
\hline 49 & 28.22206 & 34.66702 & 3 & \multirow{2}{*}{ Sharm al Majwah } \\
\hline 50 & 28.20022 & 34.66212 & 5 & \\
\hline 51 & 28.16421 & 34.65891 & 2 & \multirow{2}{*}{ Ra's ash Shaykh Humayd } \\
\hline 52 & 28.15162 & 34.61545 & 3 & \\
\hline 53 & 28.14547 & 34.61133 & 4 & \\
\hline 54 & 28.13941 & 34.60188 & 3 & \\
\hline 55 & 28.12841 & 34.59019 & 3 & \\
\hline 56 & 28.11979 & 34.58235 & 3 & \\
\hline 57 & 28.10244 & 34.57655 & 4 & \\
\hline 58 & 28.09352 & 34.57421 & 4 & \\
\hline 59 & 28.08616 & 34.58070 & 4 & \\
\hline 60 & 28.09058 & 34.58930 & 4 & \\
\hline & & & \multicolumn{1}{|c|}{} \\
\hline
\end{tabular}

Samples were analyzed using Perkin Elmer Elan 900 ICP-MS. Data processing is through ACME proprietary software.

\section{Results and discussion}

The results of this investigation can be pointed out in the followings:

1. $\mathrm{Au}, \mathrm{Be}, \mathrm{Fe}, \mathrm{Ni}$, and $\mathrm{Zn}$ have concentration values above the detection limits in only a few sea water samples. Except $\mathrm{Zn}$, the concentrations of these elements are very close to the detection limits. The locations and concentration values of these elements are presented in table 3.

2. As, Mo, $\mathrm{Rb}$, and $\mathrm{Sr}$, are found in trace amounts in all samples. The locations and concentration values of these elements are presented in table 4.

3. The major sea water constituents found in this investigation are $\mathrm{B}, \mathrm{Ba}$, $\mathrm{Br}, \mathrm{Ca}, \mathrm{Cl}, \mathrm{K}, \mathrm{Li}, \mathrm{Mg}, \mathrm{Na}$, and $\mathrm{S}$. The elements are detected in all samples with concentration values above the detection limits. The major sea water constituents in this investigation are those that represent greater than one percent of the total molar weight of the constituents of slandered sea water. Those elements are shown in table 4.

4. Fifty one elements were not found in all samples. The measured concentrations of these elements were below the detection limits. Table 5 lists these elements with their detection limits in each sample with their detection limits. 
Table 3: $\quad$ Elements that were found in some samples.

\begin{tabular}{|c|c|c|c|c|c|}
\hline & $\mathrm{Au}$ & $\mathrm{Be}$ & $\mathrm{Fe}$ & $\mathrm{Ni}$ & $\mathrm{Zn}$ \\
\hline & $\mathrm{Ppb}$ & $\mathrm{Ppb}$ & $\mathrm{ppm}$ & $\mathrm{Ppb}$ & $\mathrm{Ppb}$ \\
\hline Detection Limit & 5 & 5 & 1 & 20 & 50 \\
\hline Sample I.D. No. & & & & & \\
\hline 5 & $<5$ & 7 & $<1$ & $<20$ & $<50$ \\
\hline 10 & $<5$ & $<5$ & $<1$ & $<20$ & 168 \\
\hline 21 & $<5$ & $<5$ & $<1$ & $<20$ & 65 \\
\hline 23 & $<5$ & $<5$ & $<1$ & $<20$ & 108 \\
\hline 24 & $<5$ & $<5$ & $<1$ & $<20$ & 52 \\
\hline 32 & $<5$ & $<5$ & $<1$ & $<20$ & 164 \\
\hline 33 & $<5$ & $<5$ & $<1$ & $<20$ & 63 \\
\hline 34 & $<5$ & $<5$ & $<1$ & $<20$ & 95 \\
\hline 35 & 11 & $<5$ & $<1$ & $<20$ & $<50$ \\
\hline 36 & 6 & $<5$ & $<1$ & $<20$ & $<50$ \\
\hline 37 & $<5$ & $<5$ & 1.256 & $<20$ & $<50$ \\
\hline 39 & $<5$ & $<5$ & 1.245 & $<20$ & $<50$ \\
\hline 46 & $<5$ & 7 & $<1$ & $<20$ & $<50$ \\
\hline 47 & $<5$ & 7 & $<1$ & $<20$ & $<50$ \\
\hline 53 & $<5$ & 7 & $<1$ & $<20$ & $<50$ \\
\hline 59 & $<5$ & $<5$ & $<1$ & 23 & $<50$ \\
\hline 60 & $<5$ & 6 & $<1$ & 21 & $<50$ \\
\hline & & & & & \\
\hline
\end{tabular}

Table 4: $\quad$ A list of elements that were found in all sea water samples.

\begin{tabular}{|c|c|c|c|c|c|c|c|}
\hline & $\mathrm{As}$ & $\mathrm{B}$ & $\mathrm{Ba}$ & $\mathrm{Br}$ & $\mathrm{Ca}$ & $\mathrm{Cl}$ & $\mathrm{K}$ \\
\hline & $\mathrm{ppb}$ & $\mathrm{ppm}$ & $\mathrm{ppb}$ & $\mathrm{Ppm}$ & $\mathrm{ppm}$ & $\mathrm{ppm}$ & $\mathrm{ppm}$ \\
\hline Detection Limit & 50 & 0.5 & 5 & 0.5 & 5 & 100 & 5 \\
\hline Sample I.D. & & & & & & & \\
\hline 1 & 86 & 5.95 & 8 & 83.3 & 511 & 21349 & 474 \\
\hline 2 & 95 & 5.33 & 14 & 82.5 & 512 & 21425 & 504 \\
\hline 3 & 82 & 5.41 & 6 & 86.0 & 510 & 22786 & 483 \\
\hline 4 & 85 & 5.34 & $<5$ & 85.4 & 498 & 23064 & 483 \\
\hline 5 & 84 & 5.43 & 8 & 88.8 & 488 & 24334 & 470 \\
\hline 6 & 90 & 5.46 & 7 & 86.8 & 514 & 24117 & 482 \\
\hline 7 & 88 & 5.23 & 6 & 90.4 & 495 & 24399 & 480 \\
\hline 8 & 91 & 5.26 & 9 & 89.9 & 509 & 24730 & 467 \\
\hline 9 & 97 & 4.84 & 11 & 90.1 & 541 & 24653 & 470 \\
\hline 10 & 92 & 5.19 & 7 & 88.5 & 485 & 23932 & 480 \\
\hline 11 & 101 & 4.77 & 12 & 86.9 & 541 & 22979 & 490 \\
\hline 12 & 99 & 4.51 & 12 & 84.5 & 538 & 22632 & 464 \\
\hline 13 & 90 & 4.88 & 6 & 83.5 & 493 & 23380 & 483 \\
\hline 14 & 107 & 4.94 & 11 & 86.9 & 513 & 23209 & 494 \\
\hline
\end{tabular}


Table 4: $\quad$ Continued.

\begin{tabular}{|c|c|c|c|c|c|c|c|}
\hline & As & $\mathrm{B}$ & $\mathrm{Ba}$ & $\mathrm{Br}$ & $\mathrm{Ca}$ & $\mathrm{Cl}$ & $\mathrm{K}$ \\
\hline & $\mathrm{ppb}$ & ppm & $\mathrm{ppb}$ & Ppm & ppm & $\mathrm{ppm}$ & ppm \\
\hline Detection Limit & 50 & 0.5 & 5 & 0.5 & 5 & 100 & 5 \\
\hline \multicolumn{8}{|l|}{ Sample I.D. } \\
\hline 15 & 101 & 4.63 & 7 & 85.1 & 482 & 22789 & 464 \\
\hline 16 & 102 & 4.67 & 7 & 83.6 & 492 & 23616 & 483 \\
\hline 17 & 117 & 5.41 & 8 & 87.0 & 533 & 22926 & 522 \\
\hline 18 & 108 & 5.17 & 7 & 84.9 & 522 & 23883 & 518 \\
\hline 19 & 107 & 5.20 & 8 & 86.5 & 542 & 23832 & 532 \\
\hline 20 & 119 & 5.08 & 8 & 90.3 & 529 & 24890 & 544 \\
\hline 21 & 114 & 5.16 & 8 & 87.9 & 526 & 24180 & 525 \\
\hline 22 & 107 & 5.24 & 6 & 85.8 & 525 & 23732 & 523 \\
\hline 23 & 110 & 4.65 & 6 & 86.2 & 496 & 23928 & 481 \\
\hline 24 & 108 & 4.79 & 8 & 86.1 & 479 & 24018 & 485 \\
\hline 25 & 105 & 4.68 & 8 & 86.6 & 477 & 24285 & 483 \\
\hline 26 & 122 & 4.934 & 11 & 88.1 & 523 & 24180 & 509 \\
\hline 27 & 117 & 4.688 & 10 & 86.5 & 506 & 23628 & 496 \\
\hline 28 & 114 & 5.016 & 8 & 87.8 & 514 & 24246 & 514 \\
\hline 29 & 110 & 4.75 & 8 & 85.2 & 515 & 24629 & 520 \\
\hline 30 & 123 & 5.78 & 8 & 83.2 & 562 & 23207 & 548 \\
\hline 31 & 113 & 4.54 & 12 & 85.2 & 487 & 23967 & 499 \\
\hline 32 & 104 & 4.59 & 9 & 83.2 & 483 & 23346 & 482 \\
\hline 33 & 108 & 4.50 & 8 & 84.4 & 478 & 23745 & 490 \\
\hline 34 & 112 & 4.94 & 12 & 87.0 & 494 & 23949 & 495 \\
\hline 35 & 109 & 5.81 & 12 & 93.4 & 544 & 23905 & 515 \\
\hline 36 & 120 & 5.31 & 9 & 93.5 & 539 & 24436 & 510 \\
\hline 37 & 127 & 5.45 & 12 & 89.1 & 525 & 24340 & 506 \\
\hline 38 & 136 & 5.37 & 13 & 95.2 & 555 & 26025 & 540 \\
\hline 39 & 134 & 5.11 & 8 & 92.2 & 545 & 25525 & 532 \\
\hline 40 & 129 & 4.77 & 6 & 88.4 & 525 & 25183 & 509 \\
\hline 41 & 134 & 4.81 & 12 & 91.3 & 542 & 25883 & 526 \\
\hline 42 & 135 & 4.63 & 8 & 90.9 & 534 & 25722 & 520 \\
\hline 43 & 134 & 5.26 & 11 & 92.5 & 540 & 25951 & 524 \\
\hline 44 & 140 & 4.35 & 9 & 90.4 & 534 & 25573 & 522 \\
\hline 45 & 132 & 4.85 & 6 & 88.7 & 527 & 24441 & 519 \\
\hline 46 & 133 & 4.67 & 10 & 90.3 & 542 & 25846 & 530 \\
\hline 47 & 121 & 4.44 & 7 & 89.0 & 528 & 24633 & 530 \\
\hline 48 & 126 & 5.77 & 10 & 88.3 & 530 & 24983 & 531 \\
\hline 49 & 135 & 4.61 & 7 & 92.1 & 541 & 25045 & 526 \\
\hline 50 & 138 & 4.38 & 8 & 86.1 & 513 & 24105 & 504 \\
\hline 51 & 148 & 4.69 & 9 & 88.3 & 533 & 25186 & 522 \\
\hline 52 & 137 & 4.75 & 8 & 89.5 & 543 & 26013 & 547 \\
\hline
\end{tabular}


Table 4: $\quad$ Continued.

\begin{tabular}{|c|c|c|c|c|c|c|c|}
\hline & $\mathrm{As}$ & $\mathrm{B}$ & $\mathrm{Ba}$ & $\mathrm{Br}$ & $\mathrm{Ca}$ & $\mathrm{Cl}$ & $\mathrm{K}$ \\
\hline & $\mathrm{ppb}$ & $\mathrm{ppm}$ & $\mathrm{ppb}$ & $\mathrm{Ppm}$ & $\mathrm{ppm}$ & $\mathrm{ppm}$ & $\mathrm{ppm}$ \\
\hline Detection Limit & 50 & 0.5 & 5 & 0.5 & 5 & 100 & 5 \\
\hline Sample I.D. & & & & & & & \\
\hline 53 & 151 & 4.78 & 9 & 95.4 & 557 & 26321 & 543 \\
\hline 54 & 151 & 4.65 & 6 & 91.9 & 556 & 25953 & 543 \\
\hline 55 & 151 & 4.31 & 10 & 89.5 & 551 & 25663 & 538 \\
\hline 56 & 143 & 4.73 & 10 & 89.8 & 536 & 25710 & 544 \\
\hline 57 & 156 & 4.31 & 5 & 88.7 & 538 & 25252 & 524 \\
\hline 58 & 143 & 4.33 & 7 & 89.2 & 540 & 25004 & 557 \\
\hline 59 & 143 & 4.40 & 9 & 83.0 & 513 & 24044 & 506 \\
\hline 60 & 158 & 4.33 & 8 & 87.6 & 541 & 24803 & 529 \\
\hline
\end{tabular}

Table 5: The rest of the elements with their concentrations that were found in all sea water samples.

\begin{tabular}{|c|c|c|c|c|c|c|c|}
\hline & $\mathrm{Li}$ & $\mathrm{Mg}$ & $\mathrm{Mo}$ & $\mathrm{Na}$ & $\mathrm{Rb}$ & $\mathrm{S}$ & $\mathrm{Sr}$ \\
\hline & $\mathrm{ppb}$ & $\mathrm{ppm}$ & $\mathrm{ppb}$ & $\mathrm{ppm}$ & $\mathrm{ppb}$ & $\mathrm{ppm}$ & $\mathrm{ppb}$ \\
\hline Detection Limit & 10 & 5 & 10 & 5 & 1 & 100 & 0.01 \\
\hline Sample I.D. & & & & & & & \\
\hline 1 & 213 & 1746 & 12 & 13604 & 138 & 1126 & 9.10 \\
\hline 2 & 276 & 1736 & 14 & 13715 & 163 & 1102 & 9.33 \\
\hline 3 & 222 & 1735 & 11 & 13600 & 133 & 1101 & 9.16 \\
\hline 4 & 201 & 1728 & 12 & 13678 & 134 & 1091 & 9.36 \\
\hline 5 & 206 & 1697 & 12 & 13713 & 131 & 1090 & 10.1 \\
\hline 6 & 219 & 1717 & 12 & 13594 & 135 & 1101 & 9.53 \\
\hline 7 & 207 & 1723 & 12 & 13315 & 130 & 1104 & 9.66 \\
\hline 8 & 216 & 1731 & $<10$ & 13720 & 134 & 1133 & 10.1 \\
\hline 9 & 259 & 1688 & 12 & 13309 & 151 & 1088 & 11.0 \\
\hline 10 & 171 & 1740 & 12 & 13575 & 131 & 1094 & 9.84 \\
\hline 11 & 232 & 1728 & 12 & 13638 & 146 & 1090 & 10.9 \\
\hline 12 & 203 & 1653 & $<10$ & 13127 & 132 & 1045 & 11.2 \\
\hline 13 & 162 & 1705 & 11 & 13764 & 131 & 1104 & 10.6 \\
\hline 14 & 199 & 1731 & 12 & 13817 & 136 & 1120 & 10.2 \\
\hline 15 & 182 & 1698 & 11 & 13689 & 133 & 1090 & 10.4 \\
\hline 16 & 178 & 1687 & 11 & 13471 & 129 & 1077 & 10.2 \\
\hline 17 & 184 & 1862 & 12 & 14849 & 145 & 1183 & 10.5 \\
\hline 18 & 180 & 1804 & 12 & 14443 & 138 & 1157 & 10.1 \\
\hline 19 & 166 & 1842 & 13 & 14613 & 143 & 1181 & 10.4 \\
\hline 20 & 182 & 1914 & 13 & 15455 & 144 & 1226 & 10.5 \\
\hline 21 & 216 & 1811 & 12 & 14191 & 142 & 1174 & 10.5 \\
\hline 22 & 183 & 1819 & 13 & 14459 & 140 & 1159 & 10.6 \\
\hline & & & & & & & \\
\hline
\end{tabular}


Table 5: $\quad$ Continued.

\begin{tabular}{|c|c|c|c|c|c|c|c|}
\hline & $\mathrm{Li}$ & $\mathrm{Mg}$ & $\mathrm{Mo}$ & $\mathrm{Na}$ & $\mathrm{Rb}$ & $\mathrm{S}$ & $\mathrm{Sr}$ \\
\hline & $\mathrm{ppb}$ & $\mathrm{ppm}$ & $\mathrm{ppb}$ & $\mathrm{ppm}$ & $\mathrm{ppb}$ & $\mathrm{ppm}$ & $\mathrm{ppb}$ \\
\hline Detection Limit & 10 & 5 & 10 & 5 & 1 & 100 & 0.01 \\
\hline Sample I.D. & & & & & & & \\
\hline 23 & 179 & 1719 & 12 & 13838 & 133 & 1094 & 10.4 \\
\hline 24 & 164 & 1701 & 10 & 13711 & 129 & 1079 & 10.3 \\
\hline 25 & 168 & 1696 & 11 & 13637 & 127 & 1082 & 10.3 \\
\hline 26 & 181 & 1718 & 11 & 13574 & 140 & 1106 & 10.2 \\
\hline 27 & 181 & 1739 & 12 & 13615 & 133 & 1101 & 10.2 \\
\hline 28 & 184 & 1804 & 11 & 13913 & 136 & 1166 & 10.5 \\
\hline 29 & 195 & 1801 & 11 & 14623 & 137 & 1170 & 10.5 \\
\hline 30 & 203 & 1895 & 13 & 14964 & 158 & 1210 & 10.1 \\
\hline 31 & 186 & 1748 & 10 & 13708 & 133 & 1113 & 10.0 \\
\hline 32 & 178 & 1675 & 11 & 13479 & 128 & 1078 & 10.1 \\
\hline 33 & 171 & 1703 & 11 & 13500 & 127 & 1073 & 9.92 \\
\hline 34 & 185 & 1730 & 12 & 13748 & 133 & 1111 & 10.1 \\
\hline 35 & 240 & 1750 & 10 & 13887 & 143 & 1073 & 9.69 \\
\hline 36 & 204 & 1734 & 14 & 13783 & 147 & 1071 & 10.0 \\
\hline 37 & 214 & 1770 & 12 & 14027 & 139 & 1092 & 9.46 \\
\hline 38 & 203 & 1769 & 12 & 13919 & 152 & 1093 & 9.93 \\
\hline 39 & 198 & 1764 & 13 & 13926 & 149 & 1092 & 9.89 \\
\hline 40 & 194 & 1746 & 11 & 13805 & 143 & 1068 & 9.64 \\
\hline 41 & 183 & 1747 & 12 & 13757 & 148 & 1082 & 9.80 \\
\hline 42 & 189 & 1756 & 13 & 13842 & 148 & 1080 & 9.82 \\
\hline 43 & 187 & 1774 & 12 & 14048 & 151 & 1102 & 10.1 \\
\hline 44 & 181 & 1730 & 14 & 13498 & 141 & 1069 & 9.68 \\
\hline 45 & 188 & 1746 & 12 & 13651 & 145 & 1084 & 9.57 \\
\hline 46 & 188 & 1720 & 12 & 13672 & 146 & 1071 & 9.86 \\
\hline 47 & 178 & 1719 & 11 & 13715 & 144 & 1064 & 9.62 \\
\hline 48 & 247 & 1733 & 12 & 13702 & 143 & 1057 & 9.63 \\
\hline 49 & 174 & 1747 & 11 & 13808 & 152 & 1095 & 10.0 \\
\hline 50 & 165 & 1700 & 12 & 13442 & 140 & 1060 & 9.72 \\
\hline 51 & 180 & 1715 & 11 & 13664 & 143 & 1055 & 9.58 \\
\hline 52 & 173 & 1749 & 12 & 13779 & 149 & 1078 & 10.2 \\
\hline 53 & 181 & 1791 & 13 & 14139 & 160 & 1112 & 10.5 \\
\hline 54 & 189 & 1754 & 13 & 13946 & 152 & 1090 & 10.1 \\
\hline 55 & 173 & 1698 & 13 & 13626 & 153 & 1045 & 10.1 \\
\hline 56 & 172 & 1756 & 12 & 13878 & 150 & 1077 & 10.0 \\
\hline 57 & 186 & 1713 & 13 & 13495 & 150 & 1064 & 10.0 \\
\hline 58 & 165 & 1729 & 14 & 13571 & 151 & 1079 & 9.92 \\
\hline 59 & 172 & 1721 & 13 & 13632 & 143 & 1082 & 9.62 \\
\hline 60 & 173 & 1729 & 12 & 13584 & 148 & 1075 & 9.60 \\
\hline & & & & & & & \\
\hline & & & & & & & \\
\hline & 13 & 1378 &
\end{tabular}




\section{Conclusion}

Sixty water samples were analyzed using ICP-MS. The results of this research can be used as a baseline data in different fields of environmental analysis and also for governmental planning and development. Concentrations of some trace elements were found in the samples. These results come from different human activities. No toxic elements were found in samples with dangerous level of concentrations. Continuous monitoring should be maintained periodically. More governmental restrictions and laws should be stated to protect this region in the future. Radioisotopes concentrations measurements in this area should start to complete the research about this region.

Table 6: A summary of all elements that are not found in the samples.

\begin{tabular}{|c|c|c|}
\hline Items & $\begin{array}{c}\text { Element } \\
\text { symbol }\end{array}$ & \begin{tabular}{|c|} 
Detection \\
Limit $(\mathrm{ppb})$
\end{tabular} \\
\hline 1 & $\mathrm{Ag}$ & 5 \\
\hline 2 & $\mathrm{Al}$ & 100 \\
\hline 3 & $\mathrm{Bi}$ & 5 \\
\hline 4 & $\mathrm{Cd}$ & 5 \\
\hline 5 & $\mathrm{Ce}$ & 1 \\
\hline 6 & $\mathrm{Co}$ & 2 \\
\hline 7 & $\mathrm{Cr}$ & 50 \\
\hline 8 & $\mathrm{Cs}$ & 1 \\
\hline 9 & $\mathrm{Cu}$ & 50 \\
\hline 10 & Dy & 1 \\
\hline 11 & $\mathrm{Er}$ & 1 \\
\hline 12 & $\mathrm{Eu}$ & 1 \\
\hline 13 & $\mathrm{Ga}$ & 5 \\
\hline 14 & $\mathrm{Gd}$ & 1 \\
\hline 15 & $\mathrm{Ge}$ & 5 \\
\hline 16 & $\mathrm{Hf}$ & 2 \\
\hline 17 & $\mathrm{Hg}$ & 10 \\
\hline 18 & Ho & 1 \\
\hline 19 & In & 1 \\
\hline 20 & $\mathrm{La}$ & 1 \\
\hline 21 & $\mathrm{Lu}$ & 1 \\
\hline 22 & $\mathrm{Mn}$ & 5 \\
\hline 23 & $\mathrm{Nb}$ & 1 \\
\hline 24 & $\mathrm{Nd}$ & 1 \\
\hline 25 & $\mathrm{P}$ & 2 \\
\hline 26 & $\mathrm{~Pb}$ & 10 \\
\hline
\end{tabular}

\begin{tabular}{|c|c|c|}
\hline 27 & $\mathrm{Pd}$ & 20 \\
\hline 26 & $\mathrm{~Pb}$ & 10 \\
\hline 27 & $\mathrm{Pd}$ & 20 \\
\hline 28 & $\operatorname{Pr}$ & 1 \\
\hline 29 & $\mathrm{Pt}$ & 1 \\
\hline 30 & $\mathrm{Re}$ & 1 \\
\hline 31 & $\mathrm{Rh}$ & 1 \\
\hline 32 & $\mathrm{Ru}$ & 5 \\
\hline 33 & $\mathrm{Sb}$ & 5 \\
\hline 34 & $\mathrm{Sc}$ & 100 \\
\hline 35 & $\mathrm{Se}$ & 400 \\
\hline 36 & $\mathrm{Si}$ & 4 \\
\hline 37 & $\mathrm{Sm}$ & 2 \\
\hline 38 & $\mathrm{Sn}$ & 5 \\
\hline 39 & $\mathrm{Ta}$ & 2 \\
\hline 40 & $\mathrm{~Tb}$ & 1 \\
\hline 41 & $\mathrm{Te}$ & 5 \\
\hline 42 & Th & 5 \\
\hline 43 & $\mathrm{Ti}$ & 1 \\
\hline 44 & $\mathrm{Tl}$ & 1 \\
\hline 45 & $\mathrm{Tm}$ & 1 \\
\hline 46 & $\mathrm{U}$ & 2 \\
\hline 47 & $\mathrm{~V}$ & 200 \\
\hline 48 & $\mathrm{~W}$ & 2 \\
\hline 49 & $Y$ & 1 \\
\hline 50 & $\mathrm{Yb}$ & 1 \\
\hline 51 & $\mathrm{Zr}$ & 2 \\
\hline
\end{tabular}




\section{Acknowledgements}

The authors are grateful to Prof. Muhammad Abdul Wahid Solaiman, Prof. Hamdi Ismail Hasanien, and Prof. Abdul Moniem Al Dogdog from King Abdul Aziz University, Faculty of Earth Science, for their support and advice during the field trip and for their kind cooperation during the course of the present study.

\section{References}

[1] Nabil A. A. AL-Shwafi, Heavy metals concentration levels in some fish species in the Red Sea and Gulf of Aden, Qatar Univ. Sci. J (2002), 22: 171-176.

[2] P. C. Mayan Kutty, Azhar A. Nomani, and T.S. Thankachan, Analyses of water samples from Jeddah sea water RO/MSF plants for carbonic pollutants, Technical Report No. SWCC (RDC)-14, November, 1991.

[3] United Nations Environment Program, Regional Seas Reports and Studies No.166, Assessment of Land-based Sources and Activities Affecting the Marine Environment in the Red Sea and Gulf of Aden, 1997.

[4] The Regional Organization for the Conservation of the Environment of the Red Sea and Gulf of Aden (PERSGA), Strategic Action Program for the Red Sea and Gulf of Aden, 1998.

[5] The Regional Organization for the Conservation of the Environment of the Red Sea and Gulf of Aden (PERSGA), Status of the Living Marine Resources in the Red Sea and Gulf of Aden Region and their Management, 2000.

[6] The Regional Organization for the Conservation of the Environment of the Red Sea and Gulf of Aden (PERSGA), Marine Environmental Protection in the Red Sea and Gulf of Aden - a New Initiative (brochure), 2001.

[7] N. Ahmad, T .J. Solaija, A. Mashiatullah, M. A. Ayub, and Samia Irshad, Determination of natural and artificial radionuclides in sea water and sediments of Arabian sea, The nucleus, 41 (1-4) 2004.

[8] Mohamed O. Saeed, Salah I. Al-Khamis, Essam S. Al-Thobaiti, Ghazi Ozair, Kither Mohammad, Saeed Al-Harthi and Abdullah Bamhair, Study on the salt density index problem in the sea water reverse osmosis plants, Saline Water Desalination Research Institute-Saudi Arabia, Technical Report No. 3805/APP 96007, May 2005.

[9] Alan R. Date, Alan L. Gray, Determination of trace elements in geological samples by inductively coupled plasma source mass spectrometry, Spectro chimica Acta Part B: Atomic Spectroscopy, Volume 40, Issues 1-2, 1978, Pages 115-122.

[10] S. Greenfield, I. L. Jones and C.T. Berry. High-pressure plasmas as spectroscopic emission sources, Analyst, 89 (1964) 713-720.

[11] R. H. Wendt and V. A. Fassel. Induction-coupled plasma spectrometric excitation source, Anal. Chem., 37 (1965) 920-2. 
[12] V. A. Fassel and R. N. Kniseley. Inductively coupled plasma optical emission spectroscopy, Anal. Chem., 46 (1974) 1110A-1111A, 1116A1120 A.

[13] A. L. Gray, Mass-spectrometric analysis of solutions using an atmospheric pressure ion source, Analyst, 100 (1975) 289-99.

[14] R. S. Houk, V. A. Fassel, G. D. Flesch, H. J Svec, A. L. Gray, and C. E. Taylor, Inductively coupled argon plasma as an ion source for mass spectrometric determination of trace elements, Anal. Chem., 52 (1980) $2283-9$.

[15] Monika Shah, and Joseph A. Caruso, Inductively coupled plasma mass spectrometry in separation techniques: Recent trends in phosphorus speciation, J. Sep. Sci., 28, 1969-1984, 2005.

[16] Maria Fernanda Gine, and Ana Paula Packer, J. Braz. Soc., Vol. 21, No. 4, 575-589, 2010.

[17] Stefan Stürup, The use of ICP-MS for stable isotope tracer studies in humans: A review, Analytical and Bioanalytical Chemistry, Volume 378, Number 2, 273-282, Jan. 2004.

[18] Diane Beauchemin, Inductively Coupled Plasma Mass Spectrometry, Anal. Chem. 2006, 78, 4111-4136.

[19] A. Georgakopoulos, J.L. Fernandez Turie, and D. Gimeno, Influence of oil facilities in sea water quality: trace element distribution near Kavala, North Aegean Sea Greece, $6^{\text {th }}$ Pan-Hellenic Geographical Congress of the Hellenic Geographical Society: 343-348 (2002).

[20] Regional Organization for the Conservation of the Environment of the Red Sea and Gulf of Aden (PERSGA), Strategic Report for Red Sea, 2002. 